\title{
Synthesis of Copper-Telluride (Cu'Te) Nanowires and its Characterization using He-Ne Laser
}

\author{
Sandeep Arya, Saleem Khan, Parveen Lehana* \\ Dept. of Physics \& Electronics, University of Jammu, Jammu, India \\ ${ }^{*}$ E-mail: pklehanajournalsegmail.com
}

\begin{abstract}
Synthesis of copper-telluride nanowires has been carried out using template-assisted electrodeposition technique. The polycarbonate template is supported on a copper substrate. The synthesized nanowires were characterized using scanning electron microscopy (SEM) and X-ray diffractometer (XRD). The SEM image has confirmed the satisfactory deposition of the CuTe nanowires having $100 \mathrm{~nm}$ diameters as expected. The investigations for the optical properties of the nanowires were carried out using He-Ne laser. The analysis of the images so obtained by a high resolution digital camera at different orientations with respect to the incident light has shown that CuTe nanowires exhibit a distinct intensity patterns as compared to the patterns obtained with the available copper thin film depositions.
\end{abstract}

\section{KEYWORDS}

Electrodeposition, CuTe nanowires, optical characterization, average intensity.

\section{INTRODUCTION}

In this modern era of science and technology, nanotechnology is the one of the most significant and promising area of research. Several researchers are exploring the areas of nanoscience in almost every field due to its unique properties and may be characterized for magnetic, chemical, mechanical, optical and electronic applications. An optical characteristic of metallic nanowires is the one of the emerging area of application of nanotechnology ultrahigh density magnetic recording, ultrafast optical switching, and microwave devices.

Researchers have developed various techniques for synthesizing nanowires that includes lithographic patterning [1], vapor transport techniques [2], template based-synthesis [3] and other synthesis techniques [4] [5]. Among these techniques, the template-assisted electrodeposition has several advantages over the other fabrication techniques. Electrodeposition technique is cost effective and moreover the growth of nanomaterials can be controlled over the properties by changing the electrolyte, $\mathrm{pH}$ value, temperature, and applied voltage. Synthesis of several metallic and semiconductors nanowires has been done successfully by electrodeposition method using alumina and polymeric track etched templates [6-12]. Besides other properties, the optical properties of the nanowires have been studied extensively by various researchers for exploring different optical characterization and analytical techniques [13-17]. The scattering properties of metallic nanowires at certain wavelength range may find its applications in photo-thermal destruction of cancer cells [18], optical antenna [19], chemical and biological sensors [20], 
cloaking [21], surface-enhanced Roman spectroscopy (SERS) [22], and aperture-less near-field optical microscopy [23]. The interaction of the light with the metallic nanowires may be investigated using scattering, transmission, and reflection based techniques. The basic investigations may be carried out to study the effect of polarization direction of the illuminating polarized light, angle of the incident illuminating light, and the geometrical shape/size of the patterns developed using nanowires.

In this paper, the intensity patterns of copper thin film and copper-telluride (CuTe) nanowires deposited on copper substrate are investigated using a stationary source of polarized He-Ne laser light. The patterns of the reflected light at different orientation were recorded using high resolution digital camera at different angles with respect to the incident light. The objective of this paper is to investigate the effect of reflected light wave from the copper-telluride nanowires and the thin film depositions.

\section{EXPERIMENTAL DETAILS}

The experiment has been divided into two parts; synthesis of CuTe nanowires and its optical characterization using the incident polarized He-Ne laser light. CuTe nanowires were synthesized by template assisted electrodeposition method [26] [27]. A simple two electrode system, as shown in Fig. 1, was used for electrodepostion of CuTe nanowires.

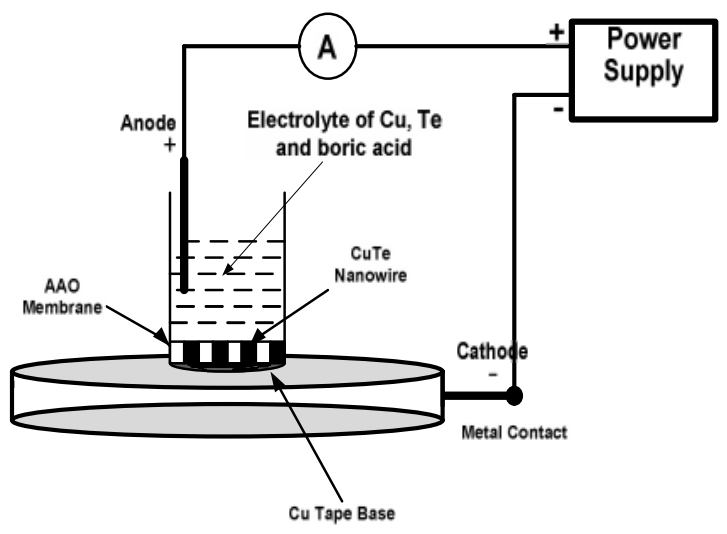

Fig.1 Schematic of two electrode cell for electrodenosition.

The copper foil acts as substrate for fabrication of CuTe nanowires. The commercially available tracketch polycarbonate membrane (whatman) of diameter $100 \mathrm{~nm}$ was used in this experiment. A limited portion of the substrate covered by membrane was selected by an "O" ring and is fixed in the top enclosure of the cell to allow it to be exposed to the electrolyte. Membrane is placed on the substrate in such a way that there exist no air bubble between the membrane and the copper substrate. The electrolytic solution was prepared in $50 \mathrm{ml}$ double distilled water containing $0.2 \mathrm{M}$ of $\mathrm{CuSO} 4.5 \mathrm{H} 2 \mathrm{O}, 0.2 \mathrm{M}$ of $\mathrm{TeO} 2$, and $0.2 \mathrm{M} \mathrm{KOH}$ at room temperature. Platinum (Pt) electrode acted as an anode for the deposition. Copper $(\mathrm{Cu})$ substrate was used as cathode for the system. Electrodeposition of CuTe nanowires was carried out by applying $0.8 \mathrm{~V}$ d.c. voltage to the electrodes. The $\mathrm{pH}$ value of the electrolytic solution was maintained between 2.5 and 3.5. The length of the CuTe nanowires were controlled by adjusting the deposition time. Finally, the samples were dried at room temperature for further characterization. Change in current density 
during the deposition is monitored very precisely. After deposition of CuTe nanowires were over, anodic aluminium oxide (AAO) membrane was dissolved in the aqueous solution of dichloromethane $\left(\mathrm{CH}_{2} \mathrm{Cl}_{2}\right)$. Finally, the sample was dried again at room temperature for further characterization.

The SEM of the CuTe nanowires deposited on the $\mathrm{Cu}$ substrate is shown in Fig.2. It shows that there is a optimum deposition of nanowires on the $\mathrm{Cu}$ substrate with uniform growth and the nanowires are vertically aligned with high aspect ratio.

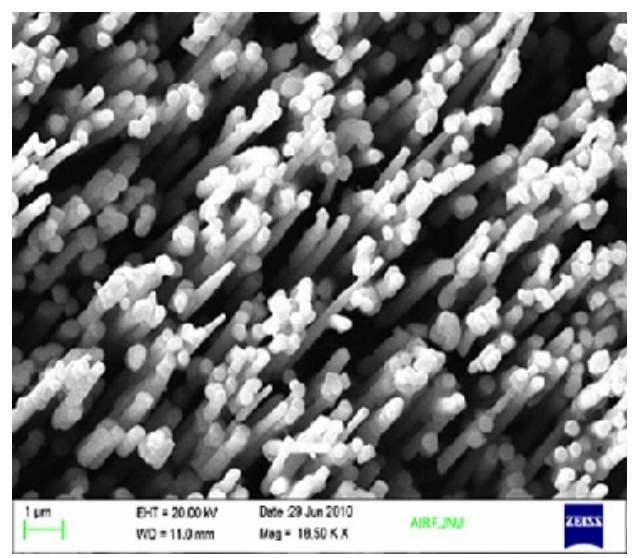

Fig. 2 SEM of CuTe nanowires deposited on $\mathrm{Cu}$ substrate.

The XRD pattern of as deposited CuTe nanowires is shown in Fig. 3. The XRD patterns were recorded in the range of scanning angle $20^{\circ}-60^{\circ}$ with a step size of $0.0170^{\circ}$ using wavelength (Ka) $1.5406 \mathrm{~A}^{\circ}$. The copper telluride $(\mathrm{CuTe})$ nanowires exist in hexagonal structural shape as the characteristic diffraction peaks in $100 \mathrm{~nm}$ pattern matches with the standard ICDD copper telluride (Cu1.75Te) data file (JCPDS Number: 45-1287) confirming the formation of copper telluride $(\mathrm{Cu} 1.75 \mathrm{Te})$ nanowires [25].

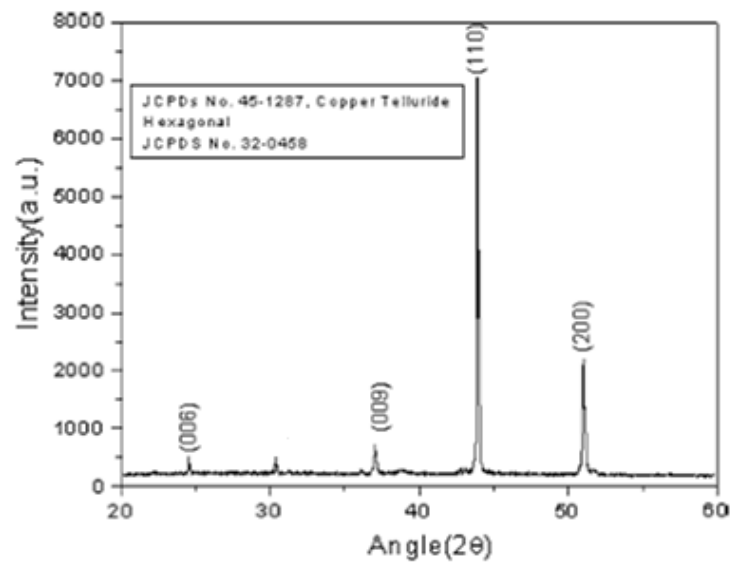

Fig. 3 XRD pattern of copper telluride nanowires $(100 \mathrm{~nm})$ on Cu substrate

The most intensive peaks in the XRD pattern indexed as (110) suggest that copper telluride pattern would have a preferential growth direction. The observed ' $\mathrm{d}$ ' value is 2.06363 for $100 \mathrm{~nm}$ 
measured at wavelength $1.54060 \AA$. The observed ' $\mathrm{d}$ ' value matches exactly with the standard value given in JCPDS (File No.45-1287) [25].

In order to investigate the intensity of copper-telluride nanowires, highly polarized He-Ne light is directed on the portion of the copper substrate where nanostructured material is deposited. Fig. 4 shows the experimental setup for determining the optical properties of $\mathrm{CuTe}$ nanowires $\mathrm{He}-\mathrm{Ne}$ light incident.

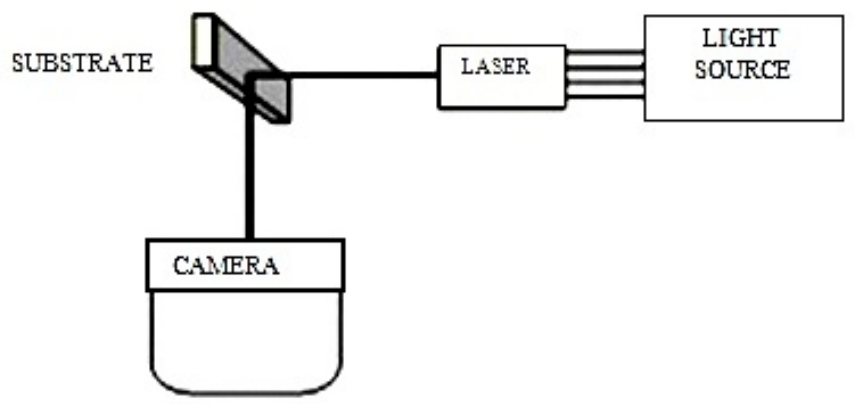

Fig. 4 Experimental setup used for determining the optical properties of CuTe nanowires

The light source is made stationary and the reflected intensity is taken as an image using a high resolution camera fixed at different angles with respect to the incident light. The He-Ne laser light source has been kept stationary at a distance of $30 \mathrm{~cm}$ from the substrate mounted on the straight platform. A high resolution digital camera is mounted on a rotating stand, so that images can be taken from different angles. The whole experiment is done in a dark room to avoid interferences from other optical sources. These images are processed using image processing toolbox from MATLAB.

\section{RESULTS \& DISCUSSIONS}

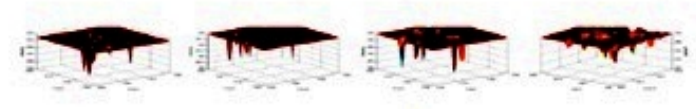

(1)

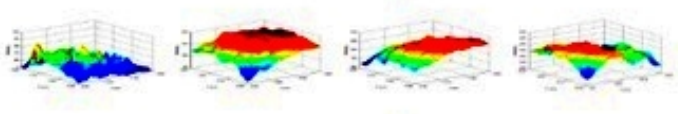

(ii)

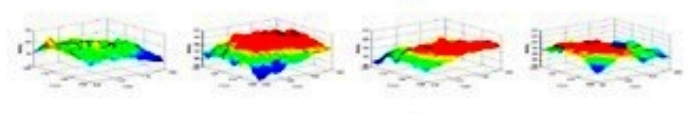

(iii)

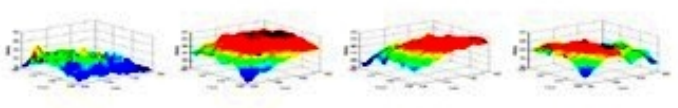

(iv)

Fig.5 3D graph of intensity of red (i), green (ii), blue (iii), and gray (iv) components over 30x30 pixel size substrate containing CuTe nanowires

The images were cut into $30 \times 30$ pixels size. Intensity of the color component is minimum at an angle of $10^{\circ}$ and maximum at $90^{\circ}$ and again starts decreasing. These images were further processed and the red, green, blue, and gray scale color components were separated using 
MATLAB. The 3D graphical image for the intensity of light on the surface of CuTe nanowires were analyzed. The color images were also converted to gray scale before plotting. The 3D images of red, green, blue, and gray scale components of the processed images at angles $30^{\circ}, 60^{\circ}$, $90^{\circ}$, and $120^{\circ}$ are shown in the Fig. 5 (i), 5 (ii), 5 (iii), and 5 (iv), respectively.

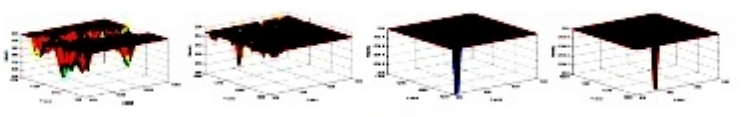

(i)

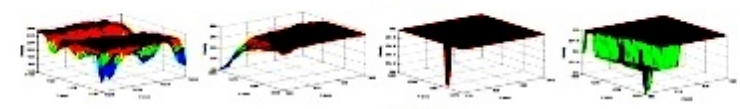

(iii)

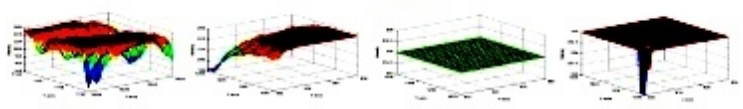

(iii)

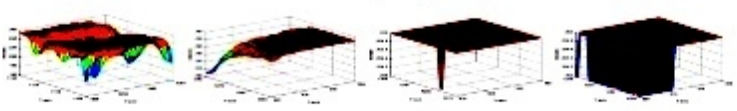

(iv)

Fig.6 3D graph of intensity of red (i), green (ii), blue (iii), and gray (iv) components over 30x30 pixel size substrate containing $\mathrm{Cu}$ substarte

Figure 6(i), 6(ii), 6(iii), and 6(iv) shows the 3D plot of intensity in pixel size with respect to angles at $30^{\circ}, 60^{\circ}, 90^{\circ}$, and $120^{\circ}$ respectively for red, green, blue and gray components for copper substrate. The average value of intensities of different color components for copper substrate is also calculated.

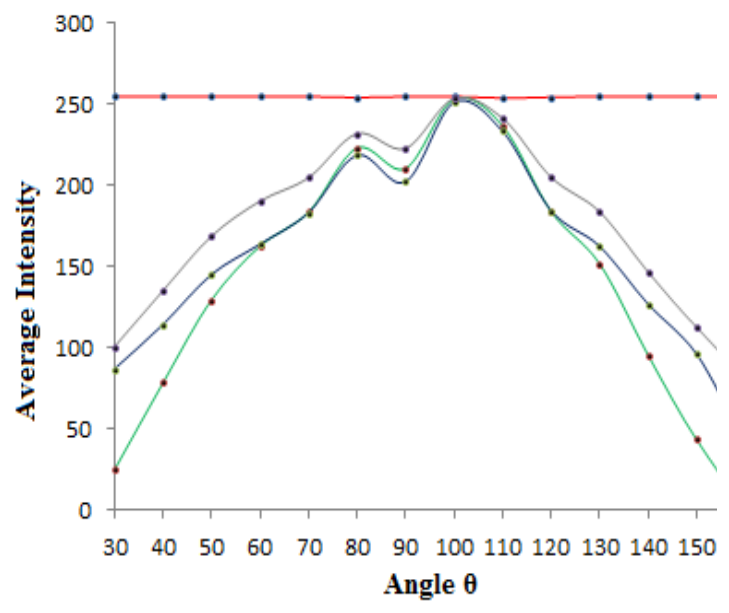

Fig.7. Average intensity of red, blue, green, and gray color components over 30x30 pixel size of $\mathrm{Cu}$ substrate containing CuTe nanowires at different angles.

The average intensity values of all the color components on 30 × 30 pixel size images were calculated and plot of these intensities with respect to angles at which they are taken is shown in 
Fig.7. The green, blue, and gray scale of the images shows similar behavior as the minima and maxima of these colors lies at same points. But red color shows different behavior i.e. average intensity remains almost same for the images taken at different angles.

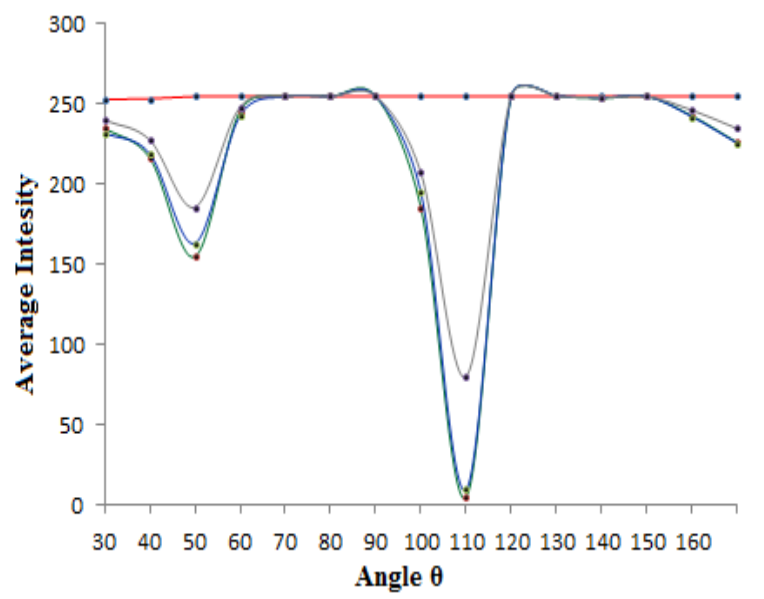

Fig.8. Average intensity of red, blue, green, and gray color components over $30 \times 30$ pixel size of $\mathrm{Cu}$ substrate at different angles.

For comparative analysis, same experiment is performed with copper substrate only. The plot between average intensity of color components at different angels is shown in Fig.8. It can be seen that the average intensity of red color component at different is same for $30 \times 30$ pixel size of the image where as for rest of the color component the average intensity is not similar.

\section{CONCLUSIONS}

In the investigation process, a polarized He-Ne light is made incident on the template assisted deposition based synthesized copper-telluride nanowires and the copper thin film deposition. The reflected light was observed through a high resolution digital camera placed at different angles with respect to the incident light. The images were resolved in red, green, blue, and grey scale components and the intensity patterns at all angles are observed. It is found that two samples behave differently with He-Ne light and show different intensity patterns at different angles. Thus, it can be concluded that change in intensity pattern with the change in angle in case of nanowires may be an important observation in the field of optical physics and nanowires can used for designing optical devices. The identification of the material from the observed pattern is on the plan for future work.

\section{REFERENCES}

[1] S. F. Abd. Rahman, U. Hashim, M. N. Md. Nor, A. M. Mohamed Nuri, M. E. A. Shohini, and S. Salleh, "Nanowire Formation Using Electron Beam Lithography," AIP Conf. Proc., Vol. 1136, pp. 504-508, 2009.

[2] S. Y. Bae, H. W. Seo, and J. H. Park, "Vertically Aligned Sulfur-Doped ZnO Nanowires Synthesized via Chemical Vapor Deposition," J. Phys. Chem. B, Vol. 108, No. 17, pp. 5206-5210, 2004.

[3] C. R. Martin, "Membrane-Based Synthesis of Nanomaterials," Chem. Mater., Vol. 8, No. 8, pp. 1739$1746,1996$.

[4] C. Xu, G. Xu, Y. Liu, and G. Wang, "A simple and novel route for the preparation of ZnO nanorods," Solid State Comm., Vol. 122, No. 3-4, pp. 175-179, 2002. 
International Journal on Organic Electronics (IJOE) Vol.1, No.2, July 2012

[5] D. Zheng, S. Sun, W. Fan, H. Yu, C. Fan, G. Cao, Z. Yin, and X. Song, "One-Step Preparation of Single-Crystalline $\beta-\mathrm{MnO}_{2}$ Nanotubes," J. Phys. Chem. B, Vol. 109, No. 34, pp. 16439-16443, 2005.

[6] Lourdes Vazquez-Gomez, SandroCattarin, Paolo Guerriero, Marco Musiani, "Preparation and electrochemical characterization of $\mathrm{Ni}+\mathrm{RuO} 2$ composite cathodes of large effective area," Electrochimica Acta, Vol. 52, No. 28, pp. 8055-8063, 2007.

[7] M. J. Zheng, L. D. Zhang, G. H. Li, W. Z. Shen, "Fabrication and optical properties of large-scale uniform zinc oxide nanowire arrays by one-step electrochemical deposition technique," Chem. Phy. Lett., Vol. 363, No. 1-2, pp. 123-128, 2002.

[8] Rosalinda Inguanta, Salvatore Piazza, Carmelo Sunseri, "Synthesis of self-standing Pd nanowires via galvanic displacement deposition," Electrochem. Comm., Vol. 11, pp. 1385-1388, 2009.

[9] M. E. Toimil Molares, V. Buschmann, D. Dobrev, R. Neumann, R. Scholz, I. U. Schuchert, J. Vetter, "Single-Crystalline Copper Nanowires Produced by Electrochemical Deposition in Polymeric Ion Track Membranes," Advanced Materials, Vol.13, Issue 1, pages 62-65, 2001.

[10] Mingliang Tian, Jinguo Wang, James Kurtz, Thomas E. Mallouk, and M. H. W. Chan, "Electrochemical Growth of Single-Crystal Metal Nanowires via a Two-Dimensional Nucleation and Growth Mechanism," Nano Letters, Vol. 3, No. 7, pp 919-923, 2003.

[11] Y. Konishi, M. Motoyama, H. Matsushima, Y. Fukunaka, R. Ishii, Y. Ito, "Electrodeposition of Cu nanowire arrays with a template," Journal of Electroanalytical Chemistry, Vol. 559, pp. 149-153, 2003.

[12] Darko Grujicic, Batric Pesic, "Electrodeposition of copper: the nucleation mechanisms," Electrochimica Acta, Vol. 47, pp. 2901-2912, 2002.

[13] S. Link, M.B. Mohamed, M.A. El-Sayed, "Simulation of the optical absorption spectra of gold nanorods as a function of their aspect ratio and the effect of the medium dielectric constant," J. Phys. Chem. B, Vol. 103, pp. 3073-3077, 1999.

[14] M. Hu, J. Chen, Z. -Y. Li, L. Au, G. V. Hartland, X. Li, M. Marquez, and Y. Xia, "Gold nanostructures: Engineering their plasmonic properties for biomedical applications," Chem. Soc. Rev, Vol.. 35, pp. 1084-1094, 2006.

[15] Z.P. Li, K. Bao, Y.R. Fang, Y.Z. Huang, P. Nordlander, H.X. Xu, "Correlation between Incident and Emission Polarization in Nanowire Surface Plasmon Waveguides," Nano Lett., Vol. 10, pp. 18311835. 2010.

[16] A.L. González, J.A. Reyes-Esqueda, C. Noguez, "Optical properties of elongated noble metal nanoparticles,” J. Phys. Chem. C, Vol. 112, pp. 7356-7362, 2008.

[17] Kursat Sendur, Ahmet Sahinoz, Eren Unlu, Serkan Yazici, and Mert Gulhan, "Near-Field Radiation from Nano-Particles and Nano-Antennas Illuminated with a Focused Beam of Light," Mater. Res. Soc. Symp. Proc. Vol. 1182 (C) 2009 Materials Research Society.

[18] J.Y. Chen, D.L. Wang, J.F. Xi, L. Au, A. Siekkinen, A. Warsen, Z.Y. Li, H. Zhang, Y.N. Xia, X.D. Li, "Immuno Gold Nanocages with Tailored Optical Properties for Targeted Photothermal Destruction of Cancer Cells," Nano Lett., Vol. 7, No. 5, pp. 1318-1322, 2007.

[19] L. Novotny, "Mapping out the resonant modes of optical antennas is part of a largely unexplored terrain - but not anymore, as a study that applies a luminescence technique to gold antennas demonstrates," Nature, Vol. 455, No. 887, 2008.

[20] Y.W.C. Cao, R.C. Jin, C.A. Mirkin, "Nanoparticles with Raman Spectroscopic Fingerprints for DNA and RNA Detection," Science, Vol. 297, No. 5586, pp. 1536-1540, 2002.

[21] J.B. Pendry, D. Schurig, D.R. Smith, “controlling electromagnetic Field,” Science, Vol. 312, No. 5781, pp. 1780-1782, 2006.

[22] H.X. Xu, E.J. Bjerneld, M. Käll, L. Börjesson, "Spectroscopy of Single Hemoglobin Molecules by Surface Enhanced Raman Scattering,” Phys Rev. Lett., Vol. 83, No. 21, pp. 4357-4360, 1999.

[23] F. Zenhausern, M. O’Boyle, and H. Wickramasinghe, "Apertureless near-field optical microscope," Appl. Phys. Lett., Vol. 65, pp. 1623-1625, 1994. 
International Journal on Organic Electronics (IJOE) Vol.1, No.2, July 2012

[24] Suresh Kumar, Virender Kundu, Anil Vohra, S. K. Chakarvarti, "Synthesis and Characterisation of Copper Telluride Nanowires via template assisted dc electrodeposition route," Journal of Material Science, Springer, 2010.

[25] ASTM Data File Nos. 45-1287, 32-0458.

[26] Parveen Lehana, Saleem Khan, and Sandeep Arya, " Fabrication And Investigations Of $\mathrm{CuFe}$ Nanowires Based Sensors," International Journal of VLSI and Signal Processing Applications, vol. 1, issue 2 , pp. 32-37, 2011.

[27] Parveen Lehana, Manpreet Kaur, Ashok Kumar, Sandeep Arya, Saleem Khan, "Effect of $\lambda$ in the EM visible region on the reflective properties of nickel nanowires," International Journal on Organic Electronics (IJOE), vol.1, no.1, pp. 13-19, 2012. 